\title{
Percepción del quehacer de enfermería en el Plan de Salud Territorial: una mirada desde enfermería ${ }^{1}$
}

Patricia Elizabeth León Saavedra²

doi:10.11144/Javeriana.IE16-2.pdqe

Cómo citar: León Saavedra PE. Percepción del quehacer de enfermería en el Plan de Salud Territorial: una mirada desde enfermeria. Invest Enferm. Imagen Desarr. 2014;16(2):13-25. doi:10.11144/Javeriana.IE16-1.pdqe

1. Artículo de investigación derivado de los resultados del proyecto titulado Percepción del rol de enfermeria en la coordinación de los planes de salud territorial, desarrollado con el apoyo financiero del Instituto de Investigaciones de la Universidad de los Llanos. Recibido: 11 de febrero de 2014. Revisado: 17 de marzo al 8 de julio de 2014. Aceptado: 4 de agosto de 2014.

2. Enfermera. Magíster en Desarrollo Educativo y Social. Magíster en Investigación en Atención Primaria. Profesora auxiliar, Facultad Ciencias de la Salud, Universidad de los Llanos, Villavicencio, Colombia. Coordinadora del grupo de investigación Favisa. Correo electrónico: pleon@unillanos.edu.co 


\section{Resumen}

Introducción: La salud en los entes territoriales es coordinada por los planes de salud territoriales (PST) que se establecen en cada municipio que propenden al mejoramiento del estado de salud de las comunidades. Objetivo: Describir la percepción del rol del profesional de enfermería que coordina el PST en el departamento del Meta, durante 2012. Material y método: Estudio cualitativo exploratorio-descriptivo, con una muestra de once profesionales de enfermería coordinadores de PST, determinada por muestreo intencional opinático. Se utilizó la entrevista semiestructurada, que fue grabada, transcrita y validada. Como herramienta para el proceso de análisis de la información se utilizó el análisis de contenido, que pretende interpretar los datos recolectados en el transcurso de la investigación en busca de los significados y sentidos que los sujetos de investigación perciben de su realidad. Resultados: Los datos se agruparon en cinco categorías de análisis: rol de enfermería, ser de enfermería, quehacer de enfermería, sentimientos y emociones en el ejercicio del rol y conocimientos acerca del ejercicio del rol. El artículo muestra los resultados de la categoria "quehacer de enfermeria", la cual agrupa cinco núcleos temáticos: el poder de decisión; la aplicación del proceso administrativo; el liderazgo; la utilización de estrategias de información, educación, comunicación, y el conocimiento y uso de los modelos teóricos de enfermería. Conclusión: Los profesionales de enfermería perciben que su quehacer en la coordinación del PST está relacionado con la coordinación de programas de promoción de salud y prevención de la enfermedad, lo cual fortalece el desarrollo de la profesión.

Palabras clave: enfermeria; percepción; liderazgo; planes y programas de salud

\section{Perception of the Practice of Nursing in the Territorial Health Care Plan: A Nursing Perspective}

\section{Abstract}

Introduction: Health in local authorities is coordinated by the territorial health plans (PST, for its initials in Spanish) established in each municipality that supports the improvement of the health status of communities. Objective: To describe the perceived role of the nurse who coordinates the PST in Meta department, in 2012.Methods: Exploratory-descriptive study, a sample of eleven professional nursing coordinators determined by deliberate opinion-based qualitative sampling. It was used a semi structured interview, which was taped, transcribed and validated. As a tool for the process of analyzing the information we used content analysis that aims at interpreting the data gathered during the research, in search of the meanings and senses the research subjects perceive from their reality. Results: The data were grouped into five categories of analysis: the role of nurses, being a nurse, the nursing job, feelings and emotions in the exercise of the role and knowledge of the exercise of the role. The paper shows results of the category "The nursing job", which groups five central themes: the power of decision; the implementation of the administrative process; leadership; strategies using information, education, communication, and knowledge and use of theoretical models of nursing. Conclusion: The nurses perceive their work in coordinating the PST is related to the coordination of programs of health promotion and disease prevention, which strengthens the development of the profession.

Keywords: nursing; perception; leadership; plans and health programs 


\section{Percepção do quefazer da enfermagem no Plano de Saúde Territorial: um olhar desde a enfermagem}

\section{Resumo}

Introdução: A saúde nos entes territoriais é coordenada pelos Planos de Saúde Territoriais (PST) estabelecidos em cada município para impulsionar o melhoramento do estado de saúde das comunidades. Objetivo: Descrever a percepção sobre o papel do profissional de enfermagem que coordena o PST no departamento de Meta, durante 2012. Material e método: Estudo qualitativo exploratório-descritivo, com amostra de onze profissionais de enfermagem coordenadores de PST, determinada por amostragem intencional baseado em opinião. Utilizou-se entrevista semiestruturada, que foi gravada, transcrita e validada. Como uma ferramenta para o processo de análise da informação foi usada análise de conteúdo, que visa interpretar os dados coletados durante a pesquisa em procura dos significados e sentidos que os sujeitos de pesquisa percebem da sua realidade. Resultados: Os dados foram agrupados em cinco categorias de análise: papel da enfermagem, ser da enfermagem, quefazer da enfermagem, sentimentos e emoções no exercício do papel e conhecimentos acerca do exercício do papel. O artigo monstra os resultados da categoria "quefazer da enfermagem", a qual está agrupando cinco núcleos temáticos: o poder de decisão; aplicação do processo administrativo; liderança; utilização de estratégias de informação, educação, comunicação, e conhecimento e uso dos modelos teóricos da enfermagem. Conclusões: Os profissionais da enfermagem percebem que o seu quefazer na coordenação do PST é relacionado com a coordenação de programas de promoção de saúde e prevenção de doença, o que fortalece o desenvolvimento da profissão.

Palavras chave: enfermagem; percepção; liderança; planos e programas de saúde 


\section{Introducción}

A partir de la Carta de Ottawa, la promoción de la salud ha ingresado a un ciclo de afianzamiento teórico-conceptual, al tiempo que se difunde como tendencia social, política e institucional, con el desarrollo de escenarios, métodos y estrategias que se expresan en las conferencias internacionales y en diversas experiencias nacionales y locales $(1,2)$.

El Sistema General de Seguridad Social en Salud (SGSSS), instituido con la Ley 100 de 1993 (3), sentó las bases para la prestación de los servicios de salud a través una red de instituciones privadas y públicas que compiten por el aseguramiento de la población, con el propósito de garantizar una eficiente atención que dé respuesta a las necesidades de la población.

Sin embargo, con el trascurso del tiempo, en busca de cumplir sus metas y principios (universalidad, equidad, calidad, progresividad, entre otros), el sistema ha sido objeto de modificaciones legales: inicialmente con la Ley 1122 de 2007 (4), que da un enfoque hacia la salud pública mediante el fortalecimiento de sus programas y a la vez establece la formulación y ejecución de los planes de salud territorial (PST) (5), fundamentados en la promoción de la salud y la prevención de la enfermedad, con el propósito de incrementar el estado de salud de la población, evitando la progresión y los desenlaces adversos de la enfermedad, enfrentando los retos del envejecimiento poblacional y la transición demográfica y disminuyendo las inequidades en salud de la población, por medio de los ejes programáticos: aseguramiento, prestación de servicios de salud, salud pública, promoción social, prevención, vigilancia y control de riesgos profesionales, emergencias y desastres.

No obstante, la Reforma 1438 de 2011 (6) busca el fortalecimiento del SGSSS a través de un modelo de prestación de servicio público en salud dentro del marco de estrategia atención primaria en salud (7), que permita la acción coordinada del Estado, las instituciones y la sociedad, para el mejoramiento de la salud, con enfoque orientado hacia la promoción de la salud y prevención de la enfermedad. Ello hace necesario que los profesionales de ciencias de la salud adquieran nuevas competencias y roles específicos que correspondan a las demandas del sistema.

Los anteriores parámetros normativos, específicamente la Ley 1122 de 2007, han incidido en las funciones que los profesionales de las ciencias de la salud deben desarrollar para responder a las nuevas exigencias del sistema, a los cambios de los perfiles epidemiológicos, a la cultura de la salud-enfermedad, al igual que al reconocimiento de sí mismo y el rol profesional de enfermería en la sociedad. Así, en el caso específico del profesional de enfermería, la práctica profesional se ha diversificado.

A fin de responder a las nuevas exigencias del actual sistema, el profesional de enfermería ha venido asumiendo distintos roles en su ejercicio laboral y ha sido parte activa en la planeación y desarrollo de actividades de promoción en salud y prevención de la enfermedad a la persona, familia y comunidad. En este campo de acción, el profesional de enfermería se ha desempeñado como coordinador de PST y ha aportado al diseño, ejecución y evaluación de actividades que favorezcan la calidad de vida de los colombianos. 
Por otra parte, el cuidado es materia de estudio de la historia de enfermería, que se enmarca en las manifestaciones del "saber" y el "quehacer" de la actividad de cuidar a lo largo de los siglos (8). Enfermería ha heredado de su proceso histórico una filosofía de responsabilidad para el cuidado de la vida humana derivada del humanismo que le da el sustento y el significado de su práctica, para lograr un grado satisfactorio de desarrollo y de salud en el ser humano (9). Así es como Nola J. Pender es reconocida por su aporte a través del modelo de promoción de la salud, al proponer que el cuidado de enfermería debe girar en torno a la promoción de la salud individual y colectiva para favorecer conductas saludables dentro de cada comunidad. Por ende, su concepción de salud parte de un componente altamente positivo, comprensivo y humanístico; toma a la persona como ser integral, al tiempo que analiza los estilos de vida, las fortalezas y las capacidades de la gente en la toma de decisiones con respecto a su salud y su vida (10).

Por otra parte, mediante procesos interpersonales, King busca entender cómo es el mundo de las personas, considerando a enfermería un medio por el cual el profesional de esta área, en el desempeño de su rol, actúa con las personas y para ellas (11). Considera, además, que la percepción es una dimensión del sistema personal y es un aspecto central de la interacción humana; por lo tanto, la percepción del profesional de enfermería y el cliente influyen en la interacción (12).

Teniendo en cuenta lo anterior, el presente artículo pretende describir la percepción del quehacer que tienen los profesionales de enfermería, como una categoría emergente resultado del proyecto Percepción del rol del profesional de enfermería que coordina planes territoriales en salud en el departamento del Meta, durante el 2012.

\section{Materiales y métodos}

Estudio cualitativo de tipo exploratorio-descriptivo en relación con el rol del profesional de enfermería que coordina el PST en municipios del departamento del Meta, desde el contexto donde se desarrollan dichos planes, lo cual constituye un referente importante en su percepción social.

La muestra estuvo conformada por once profesionales de enfermería que coordinan el PST en los municipios del departamento del Meta, la cual fue establecida por el tipo de muestreo intencional opinático y determinada por el criterio estratégico personal de voluntariedad para participar en el estudio. A cada participante se le informó sobre el estudio, y después de solicitarle su participación voluntaria, previo consentimiento informado, se programaron los encuentros para la entrevista semiestructurada. Esta contempló aspectos como: grado de desarrollo profesional, ejercicio del rol, preferencia de función específica, actividades desempeñadas, grado de satisfacción personal y valoración profesional. Las entrevistas fueron grabadas, trascritas y validadas con los sujetos de investigación.

Se tuvieron en cuenta las consideraciones éticas estipuladas para el desarrollo de procesos de investigación con seres humanos según la Resolución 8430 de 1993 del Ministerio de Salud de Colombia. La participación 
de los profesionales de enfermería estuvo bajo el anonimato: se sustituyeron por códigos los nombres de los participantes y de los municipios.

Se construyó una base de datos con la información básica (nombres, municipio y datos de contacto, como teléfono y correo electrónico) de los profesionales de enfermería que cumplieron con los criterios de inclusión de ser coordinadores del PST en los municipios del departamento del Meta durante el segundo semestre del 2012 y que aceptaran participar de la investigación.

A pesar de la infinidad de conceptos que pueden existir de diferentes autores, la percepción que tiene cada enfermero de lo que es su profesión es muy personal; por lo tanto, aunque con algunas similitudes, son muy individuales conceptos esenciales para la comprensión de la enfermería. Estos fueron estudiados por Imogene King (13), quien hace hincapié en los procesos interpersonales, dentro de los cuales contempla que la percepción es una dimensión del sistema personal y es un aspecto central de la interacción humana.

Por ello se utilizó la entrevista semiestructurada, dentro de la cual se incluyeron las siguientes preguntas orientadoras: ¿cree usted que el perfil para desempeñar la coordinación de la PST deba ser liderado por un profesional de enfermeria? ¿En su quehacer hay algunos roles que no son propios del profesional de enfermería? Estas originaron la categoría emergente quehacer de enfermeria.

Los datos recolectados albergan un contenido de conocimientos de diversos aspectos relacionados con la percepción del rol de enfermería en la coordinación de los PST en los municipios del departamento del Meta. Por ello, se utilizó como herramienta el análisis de contenido. Este es un análisis cualitativo cuya pretensión es interpretar los datos recolectados en el transcurso de la investigación en busca de los significados y sentidos que los sujetos de investigación perciben de su realidad (1).

El proceso de análisis de contenido fue realizado en dos momentos: uno sintáctico, que presenta los datos una vez segmentados como unidades de registros de acuerdo con la categoría central, y otro, en el que se agrupan los datos según pertinencia y relación entre los contenidos de las unidades (14).

El análisis de contenido se desarrolló a partir de las unidades de registro, que para esta investigación fueron las frases. Los textos fueron segmentados según los criterios de sintaxis y semántica de cada uno de ellos, para luego ser agrupados en unidades léxicas y temáticas de acuerdo con la categoría correspondiente al rol del enfermero en la coordinación del PST, de donde emerge la categoría de análisis denominada quehacer de enfermería, la cual origina el presente artículo.

\section{Objetivo}

Describir la percepción del rol del profesional de enfermería que coordinaba el PST en los municipios del departamento del Meta durante el 2012. 


\section{Resultados y discusión}

La categoría emergente quehacer de enfermería se percibe como las actividades o tareas que debe ejecutar el profesional de enfermería en la coordinación del PST en los municipios del departamento del Meta. Dentro de esta categoría se pudieron identificar cinco núcleos temáticos que revisten importancia estratégica para el alcance de los resultados deseados en las actividades de la coordinación. Estos núcleos se pueden observar en la figura 1.

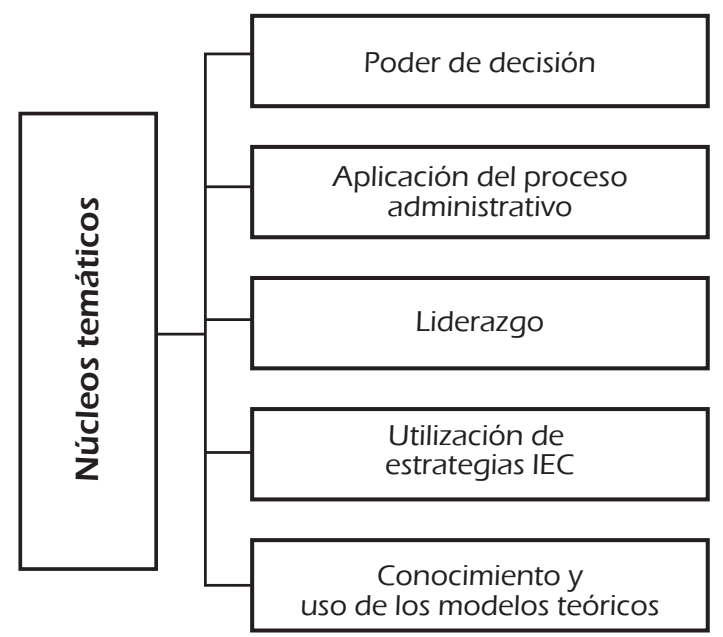

Figura 1. Categoría quehacer de enfermería de los profesionales de enfermería que coordinan planes de salud territorial en el departamento del Meta (Villavicencio, 2013)

Fuente: elaboración propia

El primer núcleo temático agrupa los datos relacionados con poder de tomar decisiones que beneficien a la comunidad en general, caracterizado con la expresiones: "administrar, me permite ser lider, coordinar, tomar decisiones, decir que hacer teniendo un conocimiento, haciendo un estudio de la situación de la comunidad" (CPSTO2QE); "se desarrollan acciones, actividades para dar soluciones ¡en lo posible... tomar decisiones!” (CPST12QE); "Nos convertimos de alguna manera en representantes de todas y cada una de las profesiones y tenemos esa capacidad de liderar, tomar decisiones" (CPST02QE); “administrar me permite ser líder, coordinar, tomar decisiones, decir que hacer teniendo un conocimiento, haciendo un estudio, teniendo un apoyo" (CPST02QE).

Sin embargo, al comparar los resultados del estudio de Astudillo Diaz (15), la toma de decisiones se presenta como una posibilidad entre los funcionarios. Ellos están conscientes de las jerarquías y líneas de mando, pero plantean en los discursos la posibilidad de mantener cierto grado de autonomía para decidir, según criterio. Eso los hace sentir que resuelven 
con sus propios medios los problemas, lo cual es percibido por los profesionales de enfermería al referir que "los profesionales de enfermería tenemos que ser esas personas políticas capaces de apoyar procesos, pero también de disentir en muchas cosas uno tiene que tener esa capacidad de manejar las dificultades y lograr que se cumplan las metas que uno ha planeado" (CPST13QE).

Por otra parte, los sujetos de estudio manifiestan que de acuerdo con las necesidades y el perfil epidemiológico de la población depende la decisión para la planeación de sus actividades; aspecto similar a la competencia disciplinar del enfermero propuesta en el proyecto educativo del programa de enfermería de la Universidad de los Llanos (16).

El segundo núcleo temático agrupa los datos percibidos por los profesionales de enfermería hacia la aplicación del proceso administrativo en el ejercicio de la coordinación, cuando refieren valorar la situación en salud, diagnosticar los problemas en salud, planificar y ejecutar acciones tendientes a mejorar el acceso y calidad en la prestación del servicio en salud, generar planes de contingencia y diseñar metas de cumplimiento. Se destaca el diagnóstico situacional como factor básico del proceso y, por tanto, fundamental para realizar todas las funciones propias de la planeación de programas y actividades del plan de salud territorial.

Con relación a los pasos del proceso administrativo: planeación, ejecución y evaluación, son tenidos en cuenta por los profesionales en su quehacer, al ser manifestados por los participantes del presente estudio, manifestado en expresiones como: "para la planeación, yo concertó las actividades con la comunidad; nos sentamos, hacemos el plan operativo anual de salud pública; hacemos también con ellos la evaluación de la ejecución de las actividades y las socializamos" (CPST12QE). "Uno puede participar desde la planeación hasta la ejecución y evaluación de toda la proyección de salud" (CPST10QE). "Coordino el plan de salud territorial, todo lo que tiene que ver con el PIC" (CPST11QE). "Planear y ejecutar todo lo que tienen que ver con salud pública con relación a las metas nacionales" (CPSTO2QE). "Hago funciones administrativas, como entrega de informes, y actividades complementarias, externas al trabajo, es decir que tengan que ver con otras dependencias, servicios públicos, SISBEN, gestión, básicamente eso" (CPST08QE). "Puede que haya unas prioridades nacionales, pero el municipio de acuerdo a su perfil de salud también debe direccionar de esa manera las actividades y es de esta manera en que se programa" (CSPTO3QE). "Igualmente tengo que responder a todo un proceso administrativo de un municipio a los diferentes entes y entidades que nos solicitan información" (CPST04QE). "Entre las actividades tenemos toda la parte de atención a la comunidad, atención al cliente, toda la parte de rendir informes, independiente hacia el área donde vaya y los procesos de evaluación y medición de indicadores de impacto, esos serian como los esenciales" (CPST05QE).

Los anteriores aspectos hacen parte de los componentes que deben realizar en los proyectos relacionados con el sector salud. Ello indica que el enfermero participa activamente en todas las etapas del proceso administra- 
tivo en el cual se planifican un conjunto de actividades que se encuentran interrelacionadas y coordinadas para alcanzar objetivos específicos dentro de los programas de promoción de la salud y prevención de la enfermedad.

De este modo, los resultados de la presente investigación son similares al estudio reflexivo de la resignificación de la gestión de los procesos de atención (17), en el cual el proceso administrativo de la gestión del profesional de enfermería posee como centro al usuario y es orientado para la asistencia, abarcando la planificación, la dirección, la evaluación de actividades desarrolladas por el personal a cargo

El tercer núcleo temático agrupa los datos que hacen referencia al liderazgo, en expresiones como: "liderar todo lo que tienen que ver con salud pública y las metas nacionales" (CPST08QE); "liderar y desarrollar lo que tiene que ver con promoción social" (CPSTO7QE); "liderar las actividades de intervenciones colectivas" (CPST02QE); "el ser líder, el llevar la vocería en lo que yo hago, en que las personas me identifiquen como eso, como la persona que está liderando” (CPST05QE); “...que me reconozcan como eso como una persona que está liderando un proceso de salud de un municipio" (CPST03QE); "uno debe tener unas cualidades, mucho liderazgo, de mucho compromiso de estudiar, de conocimiento, de la politica, de aprender a manejar cualquier tipo de situaciones" (CPST13QE).

El liderazgo, según Rozo y Abaunza (18), es el proceso de dirigir e influir en las actividades laborales de los miembros de un grupo y se reconoce como un componente fundamental que influye en la efectividad general de una organización. Desde la coordinación, el profesional de enfermería ejerce sus acciones liderazgo cuando dirige su labor hacia el cuidado del individuo o hacia el colectivo, por ejemplo: liderar los planes de emergencia municipales o llevar a cabo una coordinación intersectorial y manejo de redes de apoyo, funciones propias tanto de un espacio institucional como comunitario, donde el profesional de enfermería debe gestionar, promover y articular las acciones y propuestas de los diferentes actores del área de influencia, cuyas labores y decisiones trascienden directa o indirectamente en el ramo de la salud del municipio. Lo anterior con el fin de asegurar que su participación se fortalezca y que se vincule en diferentes momentos del desarrollo de programas de salud, incidiendo abierta y positivamente en el avance de ellos.

Lo anterior refleja la similitud que existe con el estudio titulado "E1 liderazgo en la visión de enfermeros líderes" (19), en el cual el liderazgo se reconoce como una habilidad esencial en la vida profesional del enfermero, pues lo capacita para comunicarse claramente con el grupo, ser capaz de señalar soluciones para los conflictos y tener iniciativa en la toma de decisiones, atributos que garantizan un desempeño satisfactorio en su trabajo, lo que demuestra la estrecha relación entre liderazgo y enfermería.

Por otra parte, las características del líder de este estudio coinciden con los resultados de la investigación de Lima Trindade (20), cuando los profesionales de enfermería caracterizan al líder como alguien que dirige, coordina, organiza y comanda, al ser responsable del equipo o de la ejecución del trabajo. 
El cuarto núcleo temático reúne los datos que expresan los procesos de socialización basados en estrategias de información, educación y comunicación, en expresiones como: "todas esas acciones que nosotros hacemos son prácticamente de educación en promoción y prevención” (CPST09QE); "Siempre usted va a terminar educando a la comunidad" (CPTSO3QE); "La educación continua a la comunidad creo que nunca la vamos a dejar de realizar" (CPTSO7QE); "Hay que saberse comunicar y esa parte es muy importante para que sea un buen cuidador usted tiene que escuchar, y tiene que saber hablar también, digamos que esa parte siento que es una habilidad en mi..." (CPST02); "Educar es un rol que yo desempeño, me permite definirme, como yo soy, enfermera” (CPST05QE); “... desde PST y la coordinación de estas actividades la parte de educación, me parece lo más importante con la comunidad" (CPST03QE); "Cuando hago el papel de educadora, más porque me toque es porque yo lo quiero hacer, porque para eso hay un profesional de apoyo" (CPST02QE); "También asumo el rol como educadora porque yo hago charlas, talleres... la parte de sensibilización con la comunidad..." (CPST04QE).

Los procesos desarrollados por el profesional de enfermería en la operatividad de los programas de promoción de la salud son estrategias utilizadas de forma transversal dentro del ejercicio de la profesión en los diferentes campos de acción, pues durante los diferentes momentos del proceso de atención, enfermería está informando, educando y comunicando constantemente al individuo, la familia y la comunidad, en el fomento de prácticas y conductas saludables para disminuir la aparición de factores de riesgo en la aparición de enfermedades.

Este aspecto se observa de forma diferente en el estudio de desempeño profesional de egresados del programa de enfermería de la Universidad de Caldas (21), en el cual en el área de educación sobresalen las actividades de información, educación y comunicación dirigidas al paciente y a la familia, para lo cual realizan planeación de los programas con base en el diagnóstico de necesidades, evaluación de planes, programas y proyectos. Es bajo el porcentaje de egresados (39\%) que realiza actividades de información, educación y comunicación dirigidas a grupos de la comunidad. Ellos argumentan que las principales causas de la no realización de estas son la incompatibilidad de funciones y las limitaciones de tiempo.

El quinto núcleo temático referencia datos relacionados con el conocimiento y aplicación de los modelos teóricos de enfermería por parte del profesional, al expresar no recordar el modelo desde su parte conceptual; pero sí lo hacen evidente durante el desempeño de su rol en expresiones como: "Yo no me acuerdo de ningún modelo teórico de enfermería" (CPST02QE); "yo creo que uno aplica de pronto los modelos que vio, pero uno no dice ay me voy a trabajar en este modelo" (CPSTO3QE); "yo creo que uno en la universidad recibe varios modelos teóricos, todos nos aportan algo, pero no hay uno como que uno diga con ese me quedo" (CPSTO4QE); "Esa parte teórica ya no la tengo entonces, ni siquiera podría decir que lo puedo mencionar, porque no recuerdo un modelo específico en este mo- 
mento ni nunca lo he trabajado, nunca lo he puesto en práctica... Se debería usar y que uno debería para fortalecer la carrera debería uno hacerlo" (CPST05QE; "Todos los modelos que uno aplica en el PIC son modelos de Enfermería” (CPST10QE).

Un aspecto considerado en el estudio es el relacionado con la adherencia de las enfermeras(os) a utilizar un modelo (22), referido como un gran desafío para la integración real entre las unidades formadoras de futuros profesionales de enfermería y los profesionales. Implica que estas actuaciones únicas, autónomas, propias del quehacer de este profesional, permitan alcanzar, de una u otra forma, a cumplir con las politicas nacionales de salud en lo que respecta a la gestión del cuidado de enfermería.

\section{Conclusiones}

El profesional de enfermería percibe el quehacer de enfermería en la coordinación de los PST como la aplicación del proceso administrativo basado en el liderazgo, el poder de decisión y la utilización de estrategias de información, educación y comunicación. Tal administración puede estar fundamentada en el no uso de modelos teóricos de enfermería. De ese modo, el quehacer del profesional de enfermería es pertinente en las actividades de cuidados a la salud que se desarrollan desde las coordinaciones de los PST en los diversos municipios del departamento del Meta.

El profesional de enfermería coordinador fortalece el desarrollo de la competencia social en su quehacer, el cual se refiere a sus relaciones con las personas, la cultura y la sociedad, reguladora de procesos para la salud y la vida. Los enfermeros expresan ser líderes y buenos comunicadores, lo que favorece sus relaciones y el cumplimiento de la competencia; además, son ellos quienes gestionan con los entes superiores los recursos necesarios para la ejecución del PST.

Finalmente, el transitar por diversos campos, entre ellos la coordinación del PST, posibilita el reconocimiento del saber y la praxis en la profesión. Cabe destacar que el rol de coordinador del PTS está inmerso en nuevo campo laboral para el profesional de enfermería, quien, de acuerdo con los resultados obtenidos en la categoría del quehacer, afirma que las funciones propias del rol están relacionadas con la coordinación de programas de promoción de salud y prevención de la enfermedad dirigidas hacia la población en cada uno de los municipios en el departamento del Meta.

\section{Conflictos de interés}

No existen.

\section{Financiación}

Contó con el apoyo financiero de la Dirección General de Investigaciones de la Universidad de los Llanos. 


\section{Referencias}

1. Bautista NP. Proceso de la investigación cualitativa: epistemología, metodología y aplicaciones. Bogotá: Manual Moderno; 2011.

2. Robledo R, Agudelo C. Aproximación a la construcción teórica de la promoción de la salud. Rev Salud Pública [internet]. 2011 [citado 2012 jul 14];13(6):1031-50. Disponible en: http://www.scielo.org.co/scielo. php?script $=$ sci_arttext\&pid=S0124-00642011000600015\&lng $=$ es.

3. Ley 100/1993, por la cual se crea el sistema de seguridad social integral y se dictan otras disposiciones (Diario Oficial $41.148 \mathrm{de}$ 23-12-1993).

4. Ley $1122 / 2007$ de 9 de enero, por la cual se hacen algunas modificaciones en el Sistema General de Seguridad Social en Salud y se dictan otras disposiciones (Diario Oficial 46.506 de 09-01-2007).

5. Fondo de Población de las Naciones Unidas (UNFPA) Colombia. Guía para la construcción de los planes de salud territoriales [internet]. [Citado 2012 oct 25]. Disponible en: http://www.unfpa.org.co/home/ unfpacol/public_htmlfile/PDF/Libro\%20PST\%20final.pdf.

6. Ley 1438/2011, por medio de la cual se reforma el Sistema General de Seguridad Social en Salud y se dictan otras disposiciones (Diario Oficial 47.957 de 19-01-2011).

7. García Ospina C, Tobón Correa O. Promoción de la salud, prevención de la enfermedad, atención primaria en salud y plan de atención básica: ¿qué los acerca? ¿qué los separa? Hacia Promoc Salud [internet]. 2000 [citado 2012 jul 27];(5):7-21. Disponible en: http:// promocionsalud.ucaldas.edu.co/downloads/Revista\%205_2.pdf.

8. Letelier M, Velandia A. Profesionalismo en enfermería: el hábito de la excelencia del cuidado. Av Enferm [internet]. 2010 [citado 2012 abr 05];28(2):145-58. Disponible en: http://www.scielo.org.co/scielo. php?script $=$ sci_arttext\&pid=S0121-45002010000200013\&lng=en .

9. Arroyo G. Humanismo en enfermería. Rev Enf IMSS [internet]. 2000 [citado 2012 abr 05];8(2):61-3. Disponible en: http://revistaenfermeria.imss.gob.mx/index.php?option=com_docman\&task=doc_ download\&gid=152\&Itemid=

10. Giraldo O, Toro R, Macías L, Valencia G, Palacio R. La promoción de la salud como estrategia para el fomento de estilos de vida saludables. Hacia Promoc Salud [internet]. 2010 [citado $2012 \mathrm{Abr}$ 22];15(1):128-43. Disponible en: http://www.scielo.org.co/scielo. php?script=sci_arttext\&pid=S0121-75772010000100010\&lng $=$ es.

11. Tomey M. Modelos y teorías en enfermería: conceptos principales y definiciones. 6a ed. Madrid: Elsevier Mosby; 2007.

12. Salazar Á, Martínez C. Un sobrevuelo por algunas teorías donde la interacción enfermera-paciente es el núcleo del cuidado. Av Enferm [internet]. 2008 [citado 2012 abr 05];26(2):107-15. Disponible en: http://www.enfermeria.unal.edu.co/revista/articulos/xxvi2_12.pdf.

13. Cutcliffe J, McKenna H, Hyrkäs K. Modelos de enfermería: aplicación a la práctica. Bogotá: Manual Moderno; 2011. 
14. Strauss S, Corbin, J. Bases de la investigación cualitativa: técnicas y procedimientos para desarrollar la teoría fundamentada. Medellin: Universidad de Antioquia; 2002.

15. Astudillo P, Alarcón A, Lema M. Protectores de estrés laboral: percepción del personal de enfermería y médicos, Temuco, Chile. Cienc Enferm [internet]. 2009 [citado 2013 may 04];15(3):11122. Disponible en: http://www.scielo.cl/scielo.php?script=sci arttext\&pid=S0717-95532009000300012\&lng=es. doi: 10.4067/ S0717-95532009000300012.

16. Universidad de los Llanos, Facultad de Ciencias de la Salud. Documento maestro Programa de Enfermería. Villavicencio; 2011.

17. Garlet E, Trindade LL, Lima MADS, Bonilha ALL. La resignificación de los procesos de gestión en los procedimientos de atención de enfermería. Revista Brasileña de Enfermería Online [Internet]. 2006; 5 (3): Disponible en: http://www.objnursing.uff.br/index.php/nursing/ article/view/613/144.

18. Rozo S, Abaunza M. Liderazgo transaccional y transformacional. Av Enferm [internet]. 2010 [citado 2013 abr 25];28(2):62-72. Disponible en: http://revistas.unal.edu.co/index.php/avenferm/article/ view/21380/22432

19. Souza L, Ferreira A, Nunes R, Veloso O, Vieira M, Ramos H. El liderazgo en la visión de enfermeros líderes. Enferm Glob [internet]. 2013 [citado 2014 may 18];12(30):268-80. Disponible en: http://revistas.um.es/eglobal/article/viewFile/154801/147291.

20. De Lima T, Coelho S, Adyles L, Biolchi T, Pires D, Schubert V. Influencia de los estilos de liderazgo del enfermero en las relaciones interpersonales del equipo de enfermeria. Enferm Glob [internet]. 2011 [citado 2013 may 04];10(22):1-9. Disponible en: http://scielo.isciii.es/scielo.php?script=sci_arttext\&pid=S169561412011000200014\&lng=es.

21. García C, López L, Osorio L, Realpe C. Desempeño profesional de los egresados del programa de enfermería de la Universidad de Caldas y su relación con la ley de seguridad social en salud y con el perfil de formación (Manizales 1995-2004). Hacia Promoc Salud [internet]. 2007 [citado 2013 abr 27];12(1):91-108. Disponible en: http://www.scielo.org.co/scielo.php?script=sci_arttext\&pid=S0121$75772007000100008 \& 1 n g=$ es.

22. Reyes J, Jara P, Merino J. Adherencia de las enfermeras/os a utilizar un modelo teórico como base de la valoración de enfermería. Cienc Enferm [internet]. 2007 [citado 2013 abr 27];13(1):45-57. Disponible en: http://www.scielo.cl/scielo. php?script=sci_arttext\&pid=S0717-95532007000100006\&lng=es. doi: 10.4067/S0717-95532007000100006. 
